\title{
The Build-up of the Colour-Magnitude Relation
}

\author{
Masayuki Tanaka ${ }^{1}$ and Tadayuki Kodama ${ }^{2}$ \\ ${ }^{1}$ Department of Astronomy, Graduate School of Science, University of Tokyo \\ ${ }^{2}$ National Astronomical Observatory of Japan
}

\begin{abstract}
We discuss the build-up of the colour-magnitude relation. The colour-magnitude relation first appears at the bright end and the faint end appears later. Interestingly, the buildup of colour-magnitude relation is delayed in low density environments. We suggest that galaxies follow the environment-dependent down-sizing evolution.
\end{abstract}

Keywords. galaxies: clusters: general, galaxies: evolution, galaxies: fundamental parameters

\section{Motivation and Observation}

Galaxy properties are known to strongly depend on environments in which galaxies reside. However, the origin of the environmental dependence is still unclear. To improve the situation, we carry out a systematic survey of galaxy clusters (Kodama et al. 2005). As part of this project, we observed CL0016 ( $\mathrm{z}=0.55)$ and RXJ0152 ( $\mathrm{z}=0.83)$.

\section{Results and Discussion}

We examine colour-magnitude diagrams (CMDs) of galaxies in cluster, group, and field environments as shown in Fig. 1. We observe the build-up of the colour-magnitude relation (CMR). The CMR first appears at the bright end and faint end appears later. This confirms 'down-sizing' in star formation. Interestingly, the build-up of the CMR is delayed in low density environments. That is, down-sizing is delayed in low density environments. We therefore suggest that galaxies follow the environment-dependent down-sizing evolution. Further details can be found in Tanaka et al. $(2005,2006)$

\section{References}

Kodama, T., et al. 2005, PASJ, 57, 309.

Tanaka, M., Kodama, T., Arimoto, N., Okamura, S., Umetsu, K., Shimasaku, K., Tanaka, I., \& Yamada, T. 2005, MNRAS, 362, 268.

Tanaka, M., Kodama, T., Arimoto, N., \& Tanaka, I. 2006, MNRAS, 365, 1392.
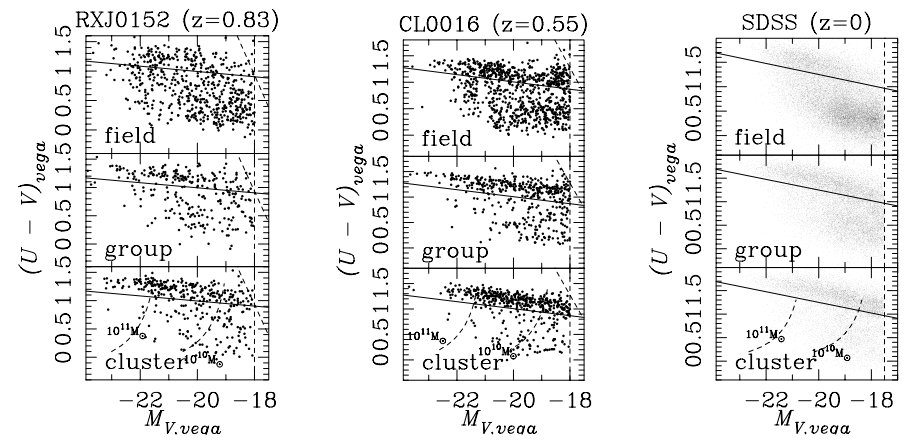

Figure 1. The rest-frame CMDs $\left(U-V\right.$ versus $\left.M_{V}\right)$ in RXJ0152 $(z=0.83)$, CL0016 $(z=0.55)$ and SDSS $(z=0)$. The solid line shows the CMR shifted blueward by 0.2 mag. 